\title{
COLONIZATION AND ANTIBIOTIC RESISTANCE DYNAMICS OF PATIENTS AT INTENSIVE CARE UNIT: OUR EXPERIENCE
}

\author{
ISHWAR CHANDRA BEHERA ${ }^{1}$, SANTOSH KUMAR SWAIN ${ }^{2}$, MAHESH CHANDRA SAHU ${ }^{3 *}$ \\ ${ }^{1}$ Department of Community Medicine, IMS and SUM Hospital, Siksha “0” Anusandhan University, Bhubaneswar, Odisha, India. \\ ${ }^{2}$ Department of Otorhinolaryngology, IMS and SUM Hospital, Siksha "O" Anusandhan University, Bhubaneswar, Odisha, India. ${ }^{3}$ Directorate \\ of Medical Research, IMS and SUM Hospital, Siksha “0” Anusandhan University, Bhubaneswar, Odisha, India. Email: mchsahu@gmail.com \\ Received: 09 January 2017, Revised and Accepted: 24 January 2017
}

ABSTRACT

Objective: Multidrug-resistant strains are developed day by day due to empirical therapy in intensive care unit (ICU). Both Gram-positive and Gramnegative strains are found in ICU are drug resistant bacteria. In this study, the prevalence of drug resistance bacteria in ICU were determined.

Methods: The clinical samples, ascitic fluid, blood, central line tip, ET aspiration pleural fluid, pus from liver abscess, sputum, tracheal aspiration, urine, and wound swabs were collected from ICU patients and cultured in specific medium. Subsequently, antibiotic sensitivity pattern of isolated bacteria was carried out with disc diffusion methods.

Results: A total number of 794 clinical samples were cultured in specific medium, and it revealed 191 of single colonies, 145 were double colonies and 87 were 3 or Multy-colonies. A total of 963 bacteria were isolated among them 180 were Gram-positive bacteria, budding yeast-like colony were 135 and rest were Gram-negative bacteria. There was no significant difference in growth of microorganisms with respect to clinical samples as t-test revealed $\mathrm{p}=0.87$. Among the Gram-negative bacteria; Klebsiela pneumonia were predominant, and Proteous mirabilis was lowest. Tigecycline and colistin were most effective antibiotics agonist all bacteria.

Conclusion: Urine samples of the patient's revealed a higher number of organisms whereas pus from liver abscess found only one number of growths. The antibiotic stewardship programs must lead to significant decrease in all antibiotic used in the ICU setting. However, this result may be required further work to determine the fruitful necessary for success

Keywords: Antibiotics, Intensive care unit, Bacterial infections, Drug resistance.

(c) 2017 The Authors. Published by Innovare Academic Sciences Pvt Ltd. This is an open access article under the CC BY license (http://creativecommons. org/licenses/by/4. 0/) DOI: http://dx.doi.org/10.22159/ajpcr.2017.v10i4.16999

\section{INTRODUCTION}

Antibiotic resistance is the most severe threats to public health today [1]. It is well documented that misuse of antibiotics contributes to develop of multidrug resistance strains and studies have shown that up to $50 \%$ of antibiotics use in clinical practice are inappropriate [2,3]. Antibiotic stewardship programs (ASPs) are highly being advised as key strategy to increase the suitability of antibiotic prescribing, with the aim of avoiding the emergence of resistance [1,3]. Some more advantage of appropriate antibiotic use results the reduction in adverse, including intensive care unit (ICU) infection and drug reactions, as well as a decrease in healthcare costs $[4,5]$. The World Health Organization (WHO), the Centers for Disease Control and Prevention along with the Infectious Diseases Society of America all endorse ASPs as an effective sources to prevent the increase and spread of antibiotic resistance $[1,6,7]$. One of the most promising ASP intervention strategies is to decrease the drug resistant strains and provide the accurate antibiotics to the ICU patients $[8,9]$. There are several studies evaluate the prospective audit and feedback programs have been conducted on wards, cabins and ICUs may be the setting with the greatest potential impact [8-10]. All ICU patients are prescribed empirical antibiotics which revealed the high levels of antibiotic resistance $[10,11]$. On the other hand, inadequate initial antibiotic therapy has been associated with mortality in critically ill patients [12]. Still, now, there have been some studies which provide the impact of audit and feedback in ICUs [11,13-16].

ICU patients are mostly immunocompromised, and they need specific drug during their treatments. ICUs are one of the sources for the containment of antibiotic resistance. Nosocomial infections are the major cause for cross contamination to seriously ill patients, and it is due to the indiscriminate use of combined antibiotics and has resulted in highly resistant bacteria pathogens [17]. Infections caused by drug-resistant microorganisms have higher morbidity and mortality rates and higher costs as compare to antibiotic sensitive bacterial infections [18-20]. The prevalence of combination antibiotic resistance in microorganism is higher among isolates from patients admitted to ICU than that among non-ICU inpatients [21-23]. Gram-negative bacteria including Pseudomonas aeruginosa have emerged as key reason for nosocomial infections and account for around $30 \%$ and $5 \%$, respectively, of all bloodstream infections [24,25]. Antibiotic resistance surveillance programs have verified an increase in resistance among these Gram-negative bacteria [23-26]. Bacterial colonization is often a primary step in the pathogenesis of nosocomial infections. Therefore, the choice of empirical antibiotic therapy depends, at least partly, on the colonization and resistance of the microorganisms. Verities of mechanisms may lead to the colonization of hospitalized patients with resistant strains $[27,28]$. The patients, after discharging from the hospital can spread the drug-resistant bacteria in the community. These resistant strains survive for a long period and performed to develop infections with resistant bacteria $[29,30]$. These studies will be limited value for major changes in antibiotic resistance of the microorganisms. Few other studies revealed that there were no any drug-resistant bacteria after discharge from the hospital [31-33]. Many studies did not identify the microorganisms for species label [30-32,34]. According to these studies, an observed increase in resistance in Gram-negative bacteria may, in fact, have been the result for verities of species of the organisms [33]. In this study, we isolate the organism from different clinical samples from the ICU patients and identifies with species label. After identification, the percentage of sensitive and resistance of antibiotics with concerned microorganisms were determined. 


\section{METHODS}

In this prospective study and approve from the Institutional Ethics Committee. The clinical samples; ascitic fluid, blood, central line tip, pleural fluid, pus from liver abscess, sputum, tracheal aspiration, urine and wound swabs were collected from the patients of neuro-ICU. All the clinical samples were cultured in suitable culture media and incubated at $37^{\circ} \mathrm{C}$ for $18 \mathrm{hrs}$. For pure culture, individually colonies were streaked in an agar plate and then processed (Fig. 1). The bacterial colony morphology was noted (Table 1) for further identifications with Gram stain and biochemical tests.

All Gram-negative bacteria were identified basing on the biochemical results (Table 2), whereas all Gram-negative bacteria were identified basing upon the test, catalase, oxidase, and coagulase results. The results are compared with the colony morphology of the culture result also. In Staphylococcus aureus golden yellow, opaque, circular colonies white butyrous consistency were observed on nutrient agar whereas, yellow colonies were observed on mannitol salt agar, and beta-hemolysis was seen on blood agar. After identification, individual bacterial were tested for antibiotic sensitivity pattern with Kirby-Bauer method. Then, the sensitive or resistances of the used antibiotics were detected by measuring the diameter of inhibitor zone created by the antibiotics (Fig. 2). All the organisms were identified basing on the previous methods [18-25].

\section{RESULTS}

A total number of 794 clinical samples from the neuro-ICU patients were cultured on suitable media. It is revealed that among them 371 did not grow on the microbiological culture media. Whereas rest samples grew in the culture media and showed single colony, double colony, and 3 or more colonies (Table 3).

There was a t-test compared with the number growth samples and number of no growth samples, and it was revealed that there was

Table 1: Morphology and culture characters of clinically isolated Gram-negative bacteria along with MTCC strains

\begin{tabular}{|c|c|c|}
\hline Bacterium & Agar media & Colony morphology \\
\hline \multirow[t]{2}{*}{ Acinetobacter baumannii } & Nutrient agar (NA) & Colorless smooth raised and pinpoint colonies \\
\hline & MacConkey (MAC) agar & Colorless smooth, raised, NLF colonies \\
\hline \multirow[t]{2}{*}{ Enterobacter aerogenes } & BA & Small, round and pin-point colony \\
\hline & MAC agar & LF and mucoid colonies \\
\hline \multirow[t]{3}{*}{ Escherichia coli } & NA & Flat dry, irregular colonies \\
\hline & MAC agar & LF, flat dry pink, irregular colonies \\
\hline & EMB agar & Flat dry, irregular colonies, with metallic green color \\
\hline \multirow[t]{2}{*}{ Klebsiella pneumoniae } & MAC agar & LF, pink, mucoid colonies \\
\hline & CLED agar & Yellow and mucoid colonies \\
\hline \multirow[t]{2}{*}{ Proteus vulgaris } & $\mathrm{BA}$ & Swarming colonies \\
\hline & CLED agar & translucent blue colonies \\
\hline \multirow[t]{3}{*}{ Pseudomonas aeruginosa } & NA & Large, irregular colonies, with bluish green pigment \\
\hline & MAC agar & Pale and yellowish (NLF), translucent without black center \\
\hline & XLD agar & Red without black center \\
\hline
\end{tabular}

NA: Not available, EMB: Eosin methylene blue agar, XLD: Xylose lysine deoxycholate, CLED: Cystine lactose electrolyte deficient medium, LF: Lactose fermenting, NLF: Non-lactose fermenting, BA: Blood agar

Table 2: Summary of results of biochemical tests of isolated Gram-negative bacteria

\begin{tabular}{lllllllllll}
\hline Bacteria & Catalase & Oxidase & Indole & MR & VP & Citrate & Urease & TSI & Nitrate & M \\
\hline A. baumannii & + & ND & - & - & + & + & V & ND & - & M \\
Enterobacter aerogenes & + & ND & - & - & + & + & V & A/A & + & M \\
Escherichia coli & + & ND & + & + & - & - & - & A/AG & + & M \\
Klebsiella oxytoca & + & ND & + & - & + & + & + & A/AG & + & NM \\
Klebsiella pneumoniae & + & ND & - & - & + & + & + & A/AG & + & NM \\
Proteus vulgaris & + & ND & - & + & - & V & + & K/A H & + & $\mathrm{M}$ \\
Proteus mirabilis & + & ND & + & + & - & V & + & K/A H & + & M \\
Pseudomonas aeruginosa & + & + & - & - & - & + & + & ND & + & M \\
\hline
\end{tabular}

+: Positive, -: Negative, V: Variable, MR: Methyl red, VP: Voges-Proskauer, TSI: Triple sugar iron, A: Acid, K: Alkali, G: Gas, $\mathrm{H}_{2} \mathrm{~S}: \mathrm{H}_{2} \mathrm{~S}$ production, M: Motile, NM: Non-motile, ND: Not done

Table 3: Types of colonies from different clinical samples of patients

\begin{tabular}{|c|c|c|c|c|c|c|}
\hline Sample & No growth & Single colony & Double colony & 3 or more colony & Total growth & Total samples \\
\hline Ascitic fluid & 14 & 2 & 0 & 0 & 2 & 16 \\
\hline Blood & 122 & 88 & 32 & 34 & 154 & 276 \\
\hline Central line tip & 1 & 4 & 0 & 0 & 4 & 5 \\
\hline ET aspiration & 2 & 2 & 0 & 0 & 2 & 4 \\
\hline Pleural fluid & 0 & 2 & 0 & 0 & 2 & 2 \\
\hline Pus from liver abscess & 7 & 1 & 0 & 0 & 1 & 8 \\
\hline Sputum & 5 & 7 & 3 & 9 & 19 & 24 \\
\hline Urine & 198 & 79 & 105 & 22 & 206 & 404 \\
\hline Wound swab & 18 & 4 & 3 & 12 & 19 & 37 \\
\hline Mean $\pm S D$ & $37.10 \pm 67.44$ & $19.10 \pm 34.05$ & $14.50 \pm 33.28$ & $8.70 \pm 11.59$ & $42.30 \pm 73.94$ & $79.40 \pm 141.02$ \\
\hline Total & 371 & 191 & 145 & 87 & & 794 \\
\hline
\end{tabular}




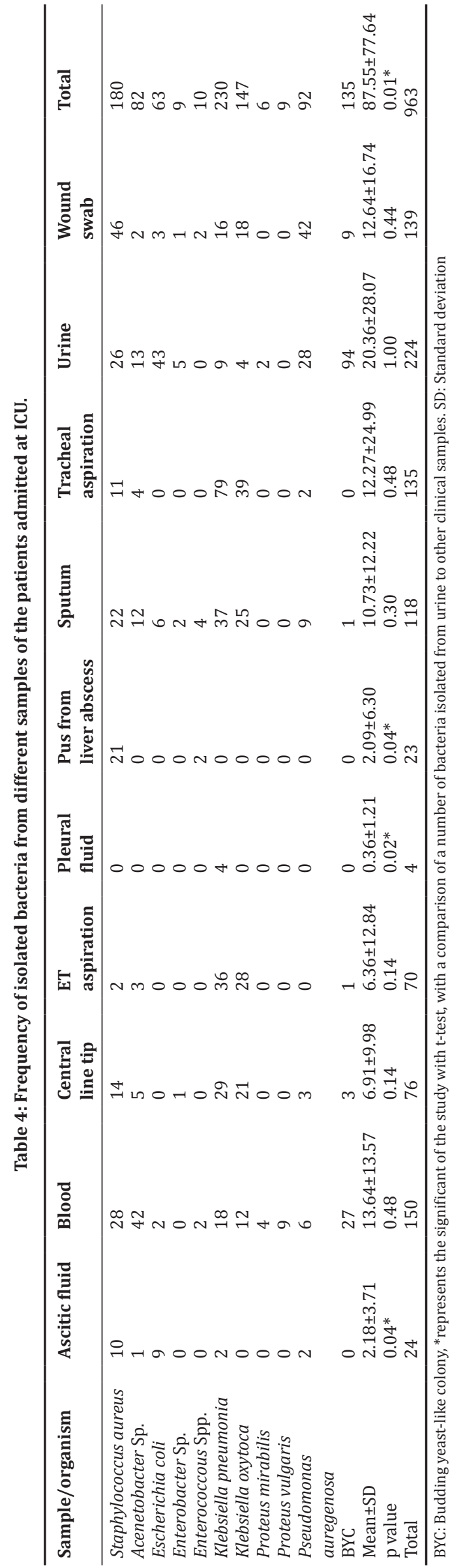

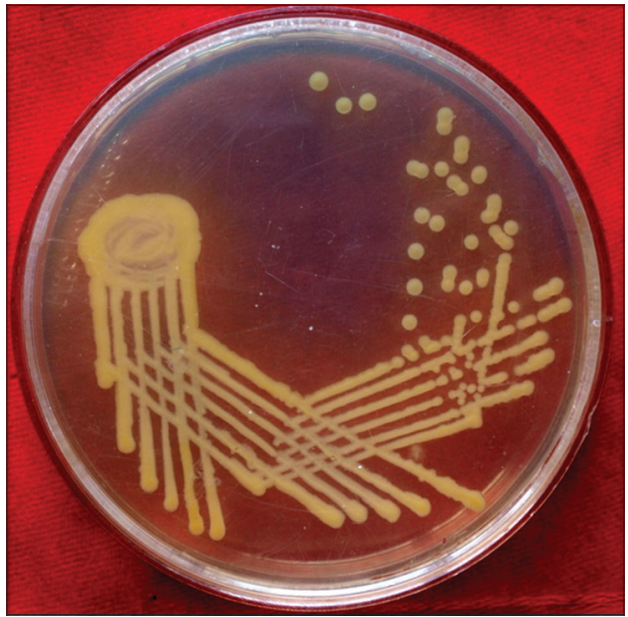

Fig. 1: Isolation of single colony with streak plate method

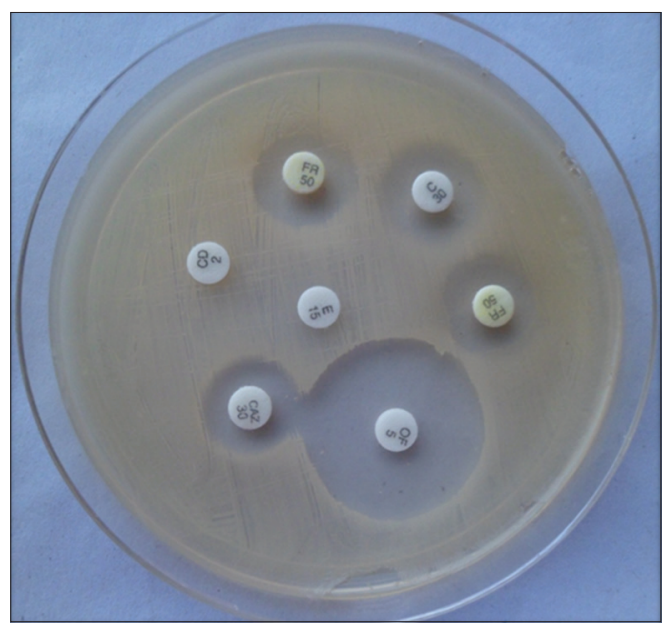

Fig. 2: Antibiotic sensitivity pattern of bacteria by disc diffusion methods

no any statistically significantly as $p=0.87$. Thus, the samples of the ICU patients may or may not contain microorganisms for growth as it is equally distributed according to t-test. All colonies were tested biochemically for the identification of bacteria. It is found that Klebsiella pneumonia (230) is the major contaminated bacteria in neuro-ICU. Minimum contaminated, i.e., 9 Proteus vulgar were isolated from the clinical samples of the neuro-ICU patients (Table 4). The organism isolated with different clinical samples were compared with t-test with urine sample and revealed significant with abscess fluid $(\mathrm{p}=0.04)$, pleural fluid (0.02), pus from liver abscess $(\mathrm{p}=0.04)$, and with total sample $(\mathrm{p}=0.01)$ (Tables 5 and 6$)$.

\section{DISCUSSION}

This study documents the microorganisms isolated from ICU and subsequently found the sensitivity and resistance of antibiotics with concerned microorganisms. The prevalence of Gram-negative bacteria is found more as compared to the Gram-positive bacteria in ICU. Sometimes, patients under treatment with antibiotics revealed suppression of growth in culture medium [35]. Hospital practice may be the cause for these cases. This hypothesis is supported by the fact that the growth of bacteria rates at different times and between the two populations were inversely related to use of antibiotic at the measured levels. Thus, the higher the level of antibiotics used, the lower the bacterial colonization rate. Second, either fresh urine specimens or some other samples were collected, and a bias may have been introduced at this point [36]. Other studies have also reported 
Table 5: Percentage of antibiotic resistance to Staphylococcus aureus

\begin{tabular}{|c|c|c|c|c|c|c|c|c|c|c|c|c|c|c|c|c|}
\hline \multirow[t]{2}{*}{ Bacteria } & \multicolumn{16}{|c|}{ Susceptibility to prescribed antibiotics } \\
\hline & AK & Ge & Ac & Am & $\mathbf{O x}$ & $\mathbf{P}$ & Ctr & Cf & of & Tei & Va & $\mathbf{E}$ & $\mathrm{Az}$ & Cd & TGC & COL \\
\hline Staphylococcus aureus & 75 & 46 & 43 & 38 & 87 & 59 & 85 & 68 & 53 & 48 & 27 & 43 & 62 & 39 & 05 & 09 \\
\hline
\end{tabular}

Table 6: Percentage of antibiotic resistance to Gram-negative bacteria

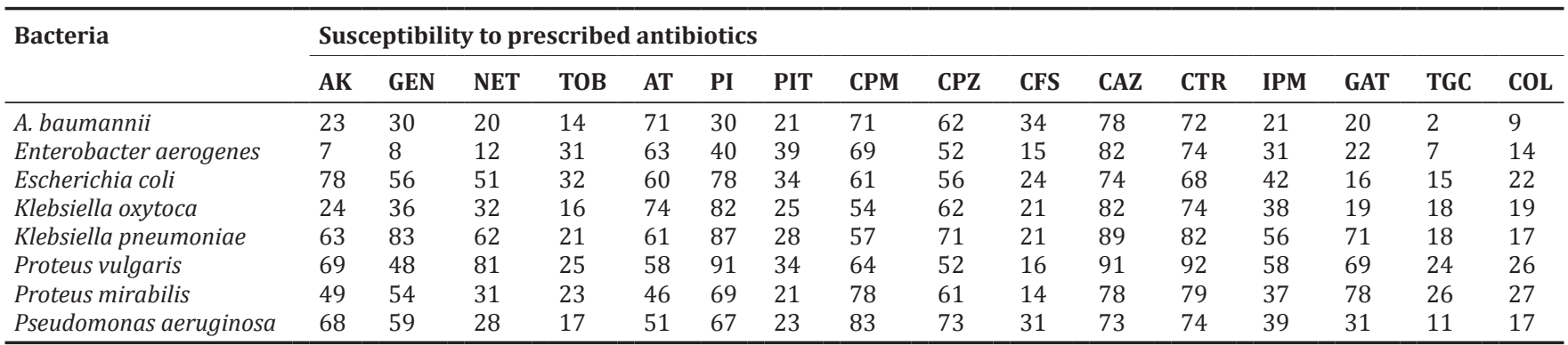

Antibiotics ( $\mu$ g/disc): AK: Amikacin 30, GEN: Gentamicin 30, NET: Netillin 30, TOB: Tobramycin 10, AT: Aztreonam 30, PI: Piperacillin 100, PIT: Piperacillin/tazobactam 100/10, CPM: Cefepime 30, CPZ: Cefoperazone 75, CFS: Cefoperazone/sulbactam 75/30, CAZ: Ceftazidime 10, CTR: Ceftriaxone 10, IPM: Imipenem 10 (carbap,

carbapenem), GAT: Gatifloxacin 30, TGC: Tigecycline 55; COL: Colistin 10

low colonization rates of Gram-negative or Gram-positive bacteria in hospitalized patients [37-39].

In a study, daily use of antibiotics was reduced $28 \%$ in the TNICU (1433 vs. 1037) but increased $14 \%$ in the MSICU (1705 vs. 1936). The total monthly antimicrobial use in the TNICU decreased by 375 DDD per 1000 patient days $(\mathrm{p}<0.0009)$ immediately following the intervention, followed by a nonsignificant downward trend in use of - 9 DDD per 1000 patient days $(\mathrm{p}=0.56)[40]$.

A high prevalence of health care-associated infections was observed, mainly caused by Gram-negative bacteria with high carbapenem resistance rates. This in combination with a high rate of antimicrobial use illustrates the urgent need to improve rational antimicrobial use and infection control efforts [41-43]. However, from a study, it was partially confirmed that ICU patients would be exposed to water amoeba-associated microorganisms and provides information about the magnitude of AAM infection in ICU patients, especially patients that have a prolonged ICU stay. However, the incidence of this exposure on the development of pneumonia remains to assess [44]. The use of antibiotics and its resistance became significant challenges for patient safety as well as management of the critically ill on ICU. ASPs aim to optimize appropriate antibiotic treatment while minimizing antibiotic resistance. Different models of ASP in ICU, include "standard" control of antibiotic prescribing such as "de-escalation strategies" through to interventional approaches utilizing biomarker-guided antibiotic prescribing. No study has demonstrated a survival benefit of ASP. Ongoing challenges to infectious disease management, reported by the WHO global report 2014, are high AMR to newer antibiotics, and regional knowledge gaps in AMR surveillance. Improved AMR surveillance data, identifying core aspects of successful ASPs that are transferable and further well-conducted trials will be necessary if ASPs are to be an effective platform for delivering desired patient outcomes and Safety through best antibiotic policy [45].

\section{CONCLUSIONS}

This study resulted the most common sources of infection on admission to the ICU. The length of hospital stay in patients with infections is longer, likely because of the increased numbers of secondary infections in these patients. However, mortality rates were identical in these groups of patients. Our results demonstrate the potential for audit and feedback to significantly reduce antimicrobial use in ICU settings.

\section{ACKNOWLEDGMENTS}

MCS is Postdoctoral Fellow of NPDF research project on "Development of molecular kit(s) to minimize the ciprofloxacin and amoxiclav resistance in chronic supportive otitis media" (grant no. PDF/2016/000772) from Department of Science and Technology, Government of India, New Delhi. We are grateful to Professor Dr. MR Nayak, President, S'OA University, Bhubaneswar, for extended facilities.

\section{REFERENCES}

1. Dubey D, Rath S, Sahu MC, Rout S, Debata NK, Padhy RN. A report on infection dynamics of inducible clindamycin resistance of Staphylococcus aureus isolated from a teaching hospital in India. Asian Pac J Trop Biomed 2013;3(2):148-53.

2. Tacconelli E. Antimicrobial use: Risk driver of multidrug resistant microorganisms in healthcare settings. Curr Opin Infect Dis 2009;22(4):352-8.

3. Dellit TH, Owens RC, McGowan JE, Gerding DN, Weinstein RA, Burke JP, et al. Infectious diseases society of America, society for healthcare epidemiology of America: Infectious diseases society of America and the society for healthcare epidemiology of America guidelines for developing an institutional program to enhance antimicrobial stewardship. Clin Infect Dis 2007;44(2):159-77.

4. Chang HT, Krezolek D, Johnson S, Parada JP, Evans CT, Gerding DN. Onset of symptoms and time to diagnosis of Clostridium difficile-associated disease following discharge from an acute care hospital. Infect Control Hosp Epidemiol 2007;28(8):926-31.

5. Shehab N, Patel PR, Srinivasan A, Budnitz DS. Emergency department visits for antibiotic-associated adverse events. Clin Infect Dis 2008;47(6):735-43.

6. Boucher HW, Talbot GH, Bradley JS, Edwards JE, Gilbert D, Rice LB, et al. Bad bugs, no drugs: No eskape! An update from the infectious diseases society of America. Clin Infect Dis 2009;48(1):1-12.

7. WHO. The Evolving Threat of Antimicrobial Resistance: Options for Action. Geneva: World Health Organization; 2012.

8. Dash A, Sahu K, Senapati JN, Debata NK, Sahoo J, Raulo BC, et al. Surveillance of antibiotic sensitivity and resistance pattern of bacteria isolated from orthopaedic wound discharge. Int J Pharm Sci Rev Res 2016;36(1):208-11

9. Lesprit P, Landelle C, Brun-Buisson C. Clinical impact of unsolicited post-prescription antibiotic review in surgical and medical wards: A randomized controlled trial. Clin Microbiol Infect 2013;19(2):E91-7.

10. Kollef MH, Micek ST. Antimicrobial stewardship programs: Mandatory for all ICUs. Crit Care 2012;16(6):179.

11. Rimawi RH, Mazer MA, Siraj DS, Gooch M, Cook PP. Impact of regular collaboration between infectious diseases and critical care 
practitioners on antimicrobial utilization and patient outcome. Crit Care Med 2013;41(9):2099-107.

12. Kumar A, Ellis P, Arabi Y, Roberts D, Light B, Parrillo JE, et al. Initiation of inappropriate antimicrobial therapy results in a fivefold reduction of survival in human septic shock. Chest 2009;136(5):1237-48.

13. Kaki R, Elligsen M, Walker S, Simor A, Palmay L, Daneman N. Impact of antimicrobial stewardship in critical care: A systematic review. J Antimicrob Chemother 2011;66(6):1223-30.

14. DiazGranados CA. Prospective audit for antimicrobial stewardship in intensive care: Impact on resistance and clinical outcomes. Am J Infect Control 2012;40(6):526-9.

15. Katsios CM, Burry L, Nelson S, Jivraj T, Lapinsky SE, Wax RS, et al. An antimicrobial stewardship program improves antimicrobial treatment by culture site and the quality of antimicrobial prescribing in critically ill patients. Crit Care 2012;16(6):R216.

16. Elligsen M, Walker SA, Pinto R, Simor A, Mubareka S, Rachlis A, et al. Audit and feedback to reduce broad-spectrum antibiotic use among intensive care unit patients: A controlled interrupted time series analysis. Infect Control Hosp Epidemiol 2012;33(4):354-61

17. Sahu MC, Dubey D, Rath S, Debata NK, Padhy RN. Multidrug resistance of Pseudomonas aeruginosa as known from surveillance of nosocomial and community infections in an Indian teaching hospital. J Public Health 2012;20(4):413-23.

18. Sahu MC, Debata NK, Padhy RN. Antibacterial activity of Argemone mexicana L. against multidrug resistant Pseudomonas aeruginosa, isolated from clinical samples. Asian Pac J Trop Biomed 2012;2(2):S800-7.

19. Sahu MC, Patnaik R, Padhy RN. In vitro combination-efficacy of ceftriaxone and leaf extract of Combretum albidum G. Don against multidrug resistant Pseudomonas aeruginosa and host-toxicity testing with human lymphocytes from human cord blood. J Acute Med 2014;4(1):26-37.

20. Sahu MC, Padhy RN. In vitro antibacterial potency of Butea monosperma Lam. against 12 clinically isolated multidrug resistant bacteria. Asian Pac J Trop Dis 2013;3(3):217-26.

21. Sahu MC, Dubey D, Rath S, Panda T, Padhy RN. Monograph: In vitro efficacy of 30 ethnomedicinal plants used by Indian aborigines against 6 multidrug resistant Gram-positive pathogenic bacteria. Asian Pac J Trop Dis 2015;5(2):136-50.

22. Sahu MC, Padhy RN. Bayesian evaluation of two conventional diagnostic methods for pathogenic fungal infections. J Acute Med 2014;4(3):109-9.

23. Sahu MC, Debata NK, Padhy RN. In vitro efficacy of Butea monosperma Lam. Against 12 clinically isolated multidrug resistant bacteria. Asia Pac J Trop Dis 2013;3(3):217-26.

24. Divyashanthi CM, Adithiyakumar S, Bharathi N. Study of prevalence and antimicrobial susceptibility pattern of bacterial isolates in a tertiary care hospital. Int J Pharm Pharm Sci 2014;7(1):185-90.

25. Jyothi P. Antibiotic sensitivity pattern of Citrobacter spp. Isolated from patients with urinary tract infections in tertiary care hospital in south India. Int J Pharm Pharm Sci 2014;7(1):252-4.

26. Fluit AC, Jones ME, Schmitz FJ, Acar J, Gupta R, Verhoef J, Antimicrobial susceptibility and frequency of occurrence of clinical blood isolates in Europe from the sentry antimicrobial surveillance program, 1997 and 1998. Clin Infect Dis 2000;30(3):454-60.

27. Pfaller MA, Jones RN, Doern GV, Kugler K. Bacterial pathogens isolated from patients with bloodstream infection: Frequencies of occurrence and antimicrobial susceptibility patterns from the sentry antimicrobial surveillance program (United States and Canada, 1997). Antimicrob Agents Chemother 1998;42(7):1762-70.
28. Bonten MJ, Weinstein RA. The role of colonization in the pathogenesis of nosocomial infections. Infect Control Hosp Epidemiol 1996;17(3): 193-200.

29. Le Gall JR, Lemeshow S, Saulnier F. A new simplified acute physiology score (SAPS II) based on a European/North American multicenter study. JAMA 1993;270(24):2957-63.

30. Austin DJ, Anderson RM. Studies of antibiotic resistance within the patient, hospitals and the community using simple mathematical models. Philos Trans R Soc Lond B Biol Sci 1999;354(1384):721-38.

31. Lipsitch M, Levin BR. The population dynamics of antimicrobial chemotherapy. Antimicrob Agents Chemother 1997;41:363-73.

32. Cooke EM, Ewins S, Shooter RA. Changing faecal population of Escherichia coli in hospital medical patients. $\mathrm{Br}$ Med J 1969;4(5683):593-5.

33. Datta N. Drug resistance and $\mathrm{R}$ factors in the bowel bacteria of London patients before and after admission to hospital. Br Med J 1969;2(5654):407-11.

34. Leistevuo T, Toivonen P, Osterblad M, Kuistila M, Kahra A, Lehtonen A, et al. Problem of antimicrobial resistance of fecal aerobic gram-negative bacilli in the elderly. Antimicrob Agents Chemother 1996;40(10):2399-403

35. Levy SB, Marshall B, Schluederberg S, Rowse D, Davis J. High frequency of antimicrobial resistance in human fecal flora. Antimicrob Agents Chemother 1988;32(12):1801-6.

36. Osterblad MA, Hakanen R, Manninen T, Leistevuo R, Peltonen O, Meurman P. A between-species comparison of antimicrobial resistance in enterobacteria in fecal flora. Antimicrob Agents Chemother 2000;44(6):1479-84

37. Shaw EJ, Datta N, Jones G, Marr FM, Froud WJ. Effect of stay in hospital and oral chemotherapy on the antibiotic sensitivity of bowel coliforms. J Hyg (Lond) 1973;71(3):529-34.

38. Sullivan A, Edlund C, Nord CE. Effect of antimicrobial agents on the ecological balance of human microflora. Lancet Infect Dis 2001;1(2):101-14

39. Bonten MJ, Nathan C, Weinstein RA. Recovery of nosocomial fecal flora from frozen stool specimens and rectal swabs: Comparison of preservatives for epidemiological studies. Diagn Microbiol Infect Dis 1997;27(4):103-6.

40. Bruinsma N, Filius PM, van den Bogaard AE, Nys S, Degener J, Endtz HP, et al. Hospitalization, a risk factor for antibiotic-resistant Escherichia coli in the community? J Antimicrob Chemother 2003;51(4):1029-32.

41. Endtz HP, den Braak NV, van Belkum A, Kluytmans JA, Koeleman JG, Spanjaard L, et al. Fecal carriage of vancomycin-resistant enterococci in hospitalized patients and those living in the community in the Netherlands. J Clin Microbiol 1997;35(12):3026-31.

42. Taggart LR, Leung E, Muller MP, Matukas LM, Daneman N. Differential outcome of an antimicrobial stewardship audit and feedback program in two intensive care units: A controlled interrupted time series study. BMC Infect Dis 2015;15(1):4.

43. Phu VD, Wertheim HF, Larsson M, Nadjm B, Dinh QD, Nilsson LE, et al. Burden of hospital acquired infections and antimicrobial use in vietnamese adult intensive care units. PloS One 2016;11(1):e0147544.

44. Bousbia S, Papazian L, Saux P, Forel JM, Auffray JP, Martin C, et al. Serologic prevalence of amoeba-associated microorganisms in intensive care unit pneumonia patients. PLoS One 2013;8(3):e58111.

45. Zhang YZ, Singh S. Antibiotic stewardship programmes in intensive care units: Why, how, and where are they leading us. World J Crit Care Med 2015;4(1):13-28. 